Original research

\title{
Stem cells from human exfoliated deciduous teeth enhance recovery from focal cerebral ischemia in rats
}

Masahiko Sugiyama $^{\text {a,1 }}$, Hisashi Hattori ${ }^{\mathrm{a}, 1,{ }^{*}}$, akanori Inoue ${ }^{\mathrm{a}}$, Hideaki Wakita ${ }^{\mathrm{b}}$, Hideharu Hibi $^{\text {a }}$, Minoru Ueda ${ }^{\text {a }}$

${ }^{a}$ Department of Oral and Maxillofacial Surgery, Laboratory Medicine, Nagoya University Graduate School of Medicine, 65 Tsurumai-cho, Shouwa-ku, Nagoya 466-8550, Japan

${ }^{\mathrm{b}}$ Department of Internal Medicine, Fujita Health University Nanakuri Sanatorium, Tsu, Japan

Article history:

Received 7 March 2013

Reeived in revised form 29 March 2013

Accepted 23 April 2013

Keywords:

Dental pulp stem cells

Neurogenesis

Cerebral ischemia

Stem cells from

${ }^{1}$ These authors contributed equally to this work.

*Corresponding author. Tel.: +81 52744 2348; fax: +81 527442352.

E-mail address: hattori6@med.na,goya-u.ac.jp (H. Hattori) 


\begin{abstract}
Purpose: The aim of this study was to investigate the effect of stem cells from human exfoliated deciduous teeth (SHED) after permanent MCAO (pMCAO).

Materials and methods: Adult male Sprague-Dawley rats were subjected to pMCAO. After pMCAO, SHED was transplanted into the brain. Motor function and infarct volume were evaluated. Neurogenesis and vasculogenesis were determined using immunochemical markers.

Results: The SHED group had more positive signals for doublecortin, neurofilament, anti-neuronal nuclei (NeuN) and rat endothelial cell antigen-1 (RECA1) in the peri-infarct area than the PBS group. Migration of doublecortin-positive neural progenitor cells (NPCs) from subventricular zone (SVZ) to the peri-infarct area was observed on day 16. Transplanted SHED merged vascular endothelial growth factor (VEGF) and stromal cell-derived factor 1 (SDF-1) positive cells.

Conclusion: SHED promoted migration and differentiation of the endogenous NPCs and induced vasculogenesis, and ameliorated ischemic brain injury after pMCAO in rats.
\end{abstract}

\title{
1. Introduction
}

Stroke is the third major cause of death worldwide and the most frequent cause of long-term disability in human beings [1]. Recently, the transplantation of bone marrow mononuclear cells achieved clinical efficacy by including angiogenesis in patients with cerebral ischemia [2,3]. However, aspiration of bone marrow to acquire bone marrow mesenchymal stem cells (BMMSCs) is an invasive and painful procedure for the donor. In addition, the number, proliferation, and differentiation potential of BMMSCs decline with increasing age [4]. In contrast, dental pulp stem cells (DPSCs) can be obtained non-invasively from teeth that are generally discarded as medical waste after extraction. DPSCs exhibit highly vasculogenic potential in vitro and promote revascularization in hind 
limb ischemia [5]. Recently, we reported that the transplanting DPSCs promoted neurogenesis and vasculogenesis in an induced peri-infarct area, and enhanced recovery after middle cerebral artery occlusion (MCAO) in rats [6]. Furthermore, these cells released growth factors, and promoted migration and differentiation of the endogenous neural progenitor cells (NPCs) in subventricular zone (SVZ). Furthermore, the proliferation rate of stem cells from human exfoliated deciduous teeth (SHED) was significantly higher than that of DPSCs and BMMSCs. SHED expressed several growth factors [7]. In the present study, we investigated the effects of SHED on neurogenesis and vasculogenesis in a rat cerebral ischemia model. In addition, we evaluated the effects of permanent MCAO (pMCAO) on the motor dysfunction and infarct volume.

\section{Materials and methods}

\subsection{Harvesting SHED}

Human dental pulp tissues were obtained from clinically healthy extracted deciduous teeth from eight patients. The Ethics Committee of Nagoya University approved our experimental protocols. SHED were isolated and cultured as previously described [8,9]. In brief, the pulp was gently removed and digested in a solution of $3 \mathrm{mg} / \mathrm{ml}$ collagenase-type I and $4 \mathrm{mg} / \mathrm{ml}$ dispase for $1 \mathrm{~h}$ at $37^{\circ} \mathrm{C}$. The cells were filtered using $70-\mu \mathrm{m}$ cell strainers (Falcon; BD Labware, Franklin Lakes, NJ), and cultured in Dulbecco's Modified Eagle Medium (DMEM; GIBCO, Rockville, MD) containing 20\% mesenchymal cell growth supplement (Lonza Inc., Walkersville, MD) and antibiotics (100 U/ml penicillin, 100 $\mathrm{mg} / \mathrm{ml}$ streptomycin, and $0.25 \mathrm{mg} / \mathrm{ml}$ amphotericin $\mathrm{B}$; GIBCO) at $37^{\circ} \mathrm{C}$ under $5 \% \mathrm{CO}_{2}$. 
After primary culture, the cells were subcultured at approximately $1 \times 10^{4}$ cells $/ \mathrm{cm}^{2}$. These cells were used in the experiments from three to five passages.

\subsection{Cerebral ischemia model}

All animal experiments were approved by the Institutional Animal Care and Use Committee (Nagoya University). Adult male Sprague-Dawley rats (Japan SLC Inc., Shizuoka, Japan) weighing 300-400 g were used. Animals were initially anesthetized with 5\% isoflurane (Abbott Laboratories, Chicago) and maintained under anesthesia with 1.5\% isoflurane in a mixture of $70 \% \mathrm{~N}_{2} \mathrm{O}$ and $30 \% \mathrm{O}_{2}$. Rectal temperature was maintained at 37 $\pm 0.5^{\circ} \mathrm{C}$ using a heating pad. Focal cerebral ischemia was induced by pMCAO [10]. A 4-0 monofilament nylon suture (Shirakawa, Tokyo, Japan) whose tip was rounded by flame heating and uniformly coated with silicone (KE-200, Shin-Etsu Chemical, Tokyo, Japan) was advanced from the external carotid artery into the internal carotid artery until it blocked the origin of MCA. The regional cerebral blood flow of MCA was measured using a laser-Doppler flowmeter (Omega FLO-N1; Omega Wave Inc, Tokyo, Japan) after occlusion. The response was considered positive and included only if the reduction in regional cerebral blood flow was greater than $70 \%$ [6].

\subsection{Cell transplantation}

Twenty-four hours after pMCAO (day 1), the rats were reanesthetized with sodium pentobarbital (Schering-Plough, Berlin, Germany) $(0.25 \mathrm{ml} / \mathrm{kg}$, intraperitoneally) and maintained under anesthesia with $1.5 \%$ isoflurane in a mixture of $70 \% \mathrm{~N}_{2} \mathrm{O}$ and $30 \% \mathrm{O}_{2}$. 
Animals were randomly divided into two groups: the SHED group $(n=4$ : day 16 sacrificed $=4)$ and the PBS group ( $n=3$ : day 16 sacrificed $=3)$. The infarction site was targeted for transplantation at the striatum with the following coordinates: $1.0 \mathrm{~mm}$ caudal to the bregma, $6.0 \mathrm{~mm}$ lateral to the midline, and $5.0 \mathrm{~mm}$ ventral to the dura (Fig. 1A, B). Subsequently, 1 $\times 10^{6}$ SHED were diluted with $2 \mu$ l of PBS and transplanted by Hamilton microsyringe (Hamilton, Reno, NV) at the third to fifth passage after labeling with 1,1-dioctadecyl-3,3,3,3-tetramethylindocarbocyanine perchlorate (DiI, Sigma) and removing all added factors into the medium. The control group received of an equal volume of PBS injected into the same site.

\subsection{Evaluation of motor disability}

Rats were blindly examined on days $1,3,6,9,12$, and 15 using a standardized motor disability scale with slight modifications [11]. Rats were scored 1 point for each of the following parameters: flexion of the forelimb contralateral to the stroke when instantly hung by the tail, extension of the contralateral hind limb when pulled from the table, and rotation to the paretic side against resistance. In addition, 1 point each was scored for circling motion to the paretic side when the rats tried to walk and for failure to exit a $50-\mathrm{cm}$ diameter circle within $10 \mathrm{~s}, 2$ points for failure to exit the circle within $20 \mathrm{~s}$, and 3 points for inability to exit the circle within 60 s. One point each was scored for the inability of the rat to extend the paretic forepaw when pushed against the table from above, laterally, and sideways. The motor disability scale was performed three times per animal per time-point. 


\subsection{Assessment of infarct volume}

Cryosections obtained from samples on day 16 were stained with hematoxylin and eosin [12]. Image J software (National Institutes of Health, ML) was used to determine each infarct area in $20-\mu \mathrm{m}$ thick ten coronal sections at 1-mm intervals. The entire infarct area was covered by these ten coronal sections. Regional infarct volumes were calculated by summing the infarct areas and multiplying them by the distance between sections $(1 \mathrm{~mm})$, followed by remediation for brain edema [13].

\subsection{Immunohistochemistry}

At day 16 after injection, the rats ware transcardially perfused with $4 \%$ paraformaldehyde solution (Nakarai Tesque, Kyoto, Japan). The brain was surgically removed, postfixed in paraformaldehyde, and immersed in $30 \%$ sucrose solution on the next day: $20-\mu \mathrm{m}$ thick coronal sections were cut on a cryostat. For immunohistochemistry, the sections were preincubated in a blocking solution (PBS containing 5\% normal serum of the species in which the secondary antibody was raised) for $2 \mathrm{~h}$ at room temperature (RT) and incubated with primary antibodies diluted for $1 \mathrm{~h}$ at RT. The primary antibodies were as follows: an NPC marker, rabbit anti-doublecortin (1:50, Abc a m In c., Ca mbr id ge, MA); a neuron marker, rabbit anti-neurofilament H (1:200, Chemicon, Temecula, CA); and mouse anti-neuronal nuclei (anti-NeuN, 1:500, Chemicon); endothelial cell marker, mouse anti- rat endothelial cell antigen 1 (RECA1, 1:50, Monosan, Uden, The Netherlands); vascular endothelial growth factor (VEGF) marker, goat anti-VEGF (VEGF [P-20]:sc-1836, 1:200, Santa Cruz Biotech, CA) ; stromal cell-derived factor 1 (SDF-1) marker, rabbit anti-SDF-1 
(SDF-1 [FL-93]:sc-28876, 1:200, Santa Cruz Biotech, CA).After washing, the sections were incubated for $1 \mathrm{~h}$ at RT with secondary antibodies for neurofilament H/doublecortin/SDF-1, donkey anti-rabbit IgG FITC (1:400; Jackson ImmunoResearch, USA); for NeuN/RECA1, goat anti-mouse IgG FITC (1:200; MP Biomedicals, LLC,Solon, OH,USA); and for VEGF, donkey anti-goat IgG- FITC (1:200; Jackson ImmunoResearch, USA). Adjacent sections were used as negative controls. In the control sections, all sections were processed in the same manner except the primary antibodies. To identify NPC migration from the SVZ, we observed the SHED and PBS cryosections group on day 16 stained with anti-doublecortin using fluorescence (BZ-9000, Keyence, Tokyo, Japan) and BZ-HIC microscopes (Keyence).

\section{7. $\quad$ Statistical analyses of the density of NPCs}

To identify migration of NPC from SVZ, we observed the cryosections on days 16 with anti-doublecortin on fluorescence microscope (BZ-9000; Keyence). The density of doublecortin in the peri-SVZ area (Fig. 6E) in the SHED and PBS groups was determined. In two groups $(n=3)$, each three sections including peri-SVZ area were stained with anti-doublecortin. The microscopic images were scanned toward the direction of elongating NPCs from SVZ, three frames $\left(0.39 \mathrm{~mm}^{2}\right)$ were measured and summed up for each section. The positively stained area relative to total area $\left(1.17 \mathrm{~mm}^{2}\right)$ was statistically analyzed using a Dynamic cell count, BZ-HIC. Data are reported as means \pm SD. $p$-Values were calculated using the unpaired Student's $t$-test.

\section{Results}

\section{1. evaluation of motor function}


The two groups (SHED group, PBS group) displayed almost same high motor-function scores at the early-stage (day 1 , scores are $8.67 \pm 0.58 ; 8.00 \pm 1.00$, and day $3,8.33 \pm 0.58$; $8.0 \pm 1.00$ ). At the middle-stage (days 6-9), differences in the score appeared gradually between the two groups (day $6,6.67 \pm 0.58 ; 7.33 \pm 1.15$, day $9,5.67 \pm 0.58 ; 7.33 \pm 1.15$ ). On day12 and day15, considerable progressive improvement was observed in motor disability in the SHED group compared with that in the PBS group (day 12, $4.67 \pm 0.58$; $7.00 \pm 1.00$, day $15,3.67 \pm 0.58 ; 6.33 \pm 1.15)($ Fig. 2$)$

\section{2. reduction of infarct volume}

As shown in Fig. 3, a significant decrease in the infarct volume was observed on day 16 in the SHED group (day $16,42.27 \pm 19.7 \mathrm{~mm}^{3}, n=4$ ) compared to that in the PBS group (day $\left.16,197.72 \pm 35.1 \mathrm{~mm}^{3}, n=3\right)$.

\subsection{Shed cell outcome and expression of neurotrophic factors}

DiI-labeled transplanted SHED was characterized by round-to-oval nuclei with minimally variable cytoplasm. Transplanted cells survived and mainly migrated from the original injection site at the center of peri-infarct area (Fig. 1C, D) in the cortex and striatum. Transplanted cells localized in the proximity of doublecortin (Fig. 4A), neurofilament (Fig. 4C), and NeuN (Fig. 4E) positive cells and RECA1-positive cells (Fig. 4G) on day 16. No evidence of SHED differentiation into neurons or endothelial cells was detected. Migration of doublecortin-positive NPCs from the SVZ to the peri-infarct area was observed on day 16. This migration was more prominent in the SHED group (Fig. 6A) than that in the PBS 
group (Fig. 6B). Many of transplanted cells merged VEGF (Fig. 5A) and SDF-1 (Fig. 5D) positive cells in the peri-infarct area (Fig. 5C, F).

\section{Discussion}

In the mammalian brain, the production of new neurons during adulthood continues in the subventricular zone (SVZ) at the lateral walls of ventricles and in the subgranular zone in the dentate gyrus of the hippocampus [14-16]. In the injured brain, NPCs migrate toward the damaged area. Within a week after the insult, NPCs in the SVZ begin to proliferate, and neuroblasts with the migratory morphology and newly generated neurons appear at the boundary of the damaged area in the striatum $[17,18]$.

In the present study, we demonstrated that transplanted SHED migrated to the peri-infarct area (Fig. 1C) and that SHED administration ameliorated ischemic tissue injury and accelerated the functional recovery after pMCAO. Transplanted SHED merged VEGF and SDF-1 in the per-infarct area. Some reports suggested that expression of VEGF stimulates neurogenesis and angiogenesis in the injured brain [19,20]. In addition, some studies reported that SDF-1 plays a neuroprotective role during the repair of neuronal tissue injury $[21,22]$. Therefore, VEGF and SDF-1 expression induced by SHED suggested that the transplanted cells also promoted angiogenesis and neuroprotection after ischemia. SHED group had more positive signals of doublecortin, neurofilament, NeuN, and RECA1 in the peri-infarct area than the PBS group. Migration of doublecortin-positive NPCs from the SVZ to the peri-infarct area was observed on day 16 . This migration was more prominent in the SHED group (Fig. 6A) than that in the PBS group (Fig. 6B). Therefore, SHED 
administration promotes migration and differentiation of the endogenous NPC, induces vasculogenesis, and ameliorates ischemic brain injury after pMCAO [6].

To apply DPSCs clinically, transplantation must first be researched using human dental pulp to identify any immunogenic problems. The last molar teeth and deciduous teeth are potential candidates for Human dental pulp stem cell source. Above all, deciduous teeth can be obtained non-invasively from teeth that are discarded as medical waste after being extracted without ethcal problem. Deciduous teeth have a high proliferation capacity and can be easily harvested [7]. If we bank stem cells from own deciduous teeth, they could be utilized in the future. Banking stem cells from an individual's own deciduous teeth is a reasonable and simple alternative to harvesting stem cells from BMMSCs [23]. Therefore, deciduous teeth are the most appropriate potential source for cell therapy of stroke.

Finally, we explored the plausible underlying mechanisms of preventing graft rejection because of SHED administration into the brain of immunocompetent rats. The blood-brain barrier plays a critical role maintaining the immune-privileged status of the central nervous system [24]. BMMSCs are not rejected by hosts and therefore, immunosuppression is not required in rodents [25]. DPSC have many similarities to mesenchymal stem cells; transplanted SHED possesses immunosuppressive properties [26].

In conclusion, transplanting SHED promoted neurogenesis and vasculogenesis in an induced peri-infarct area, and enhanced recovery after pMCAO in rats. However, further research is required to understand the underlying mechanisms of this event. For potential clinical application and translational studies, the safety of SHED must be assessed, including tumor formation. Regeneration therapy using SHED is a potential therapy for 
stroke.

\section{Acknowledgments}

We thank Mrs. Mami Naruse for her assistance. This work was supported by funds from Collaborative Development of Innovative Seeds, potentiality verification stage from Japan

Science and Technology Agency, and a Grant-in-Aid for Scientific Research from the Ministry of Education, Science, Sports and Culture, Japan, \#22890082 (M.S.).

\section{References}

1. Donnan GA, Fisher M, Macleod M, Davis SM. Stroke. Lancet 2008;371:1612-23.

2. Honmou O, Houkin K, Matsunaga T, Niitsu Y, Ishiai S, Onodera R, et al. Intravenous administration of auto serum-expanded autologous mesenchymal stem cells in stroke. Brain 2011;134:1790-807.

3. Bang OY, Lee JS, Lee PH, Lee G. Autologous mesenchymal stem cell transplantation in stroke patients. Ann Neurol. 2005;57:874-82.

4. Kern S, Eichler H, Stoeve J, Klüter H, Bieback K. Comparative analysis of mesenchymal stem cells from bone marrow, umbilical cord blood, or adipose tissue. Stem Cells 2006;24:1294-301.

5. Iohara K, Zheng L, Wake H, Ito M, Nabekura J, Wakita H, et al. A novel stem cell source for vasculogenesis in ischemia: subfraction of side population cells from dental pulp. Stem Cells 2008;26:2408-18.

6. Sugiyama M, Iohara K, Wakita H, Hattori H, Ueda M, Matsushita K, et al. Dental pulp derived $\mathrm{CD} 31^{-} / \mathrm{CD} 146^{-}$side population stem/progenitor cells enhance recovery of focal cerebral ischemia in rats. Tissue Eng Part A 2011;17:1303-11.

7. Nakamura S, Yamada Y, Katagiri W, Sugito T, Ito K, Ueda M. Stem cell proliferation pathways comparison between human exfoliated deciduous teeth and dental pulp stem cells by gene expression profile from promising dental pulp. J Endod 2009;35:1536-42. 
8. Gronthos S, Mankani M, Brahim J, Robey PG, Shi S. Postnatal human dental pulp stem cells (DPSC) in vitro and in vivo. Proc Natl Acad Sci U S A 2000;97:13625-30.

9. Miura M, Gronthos S, Zhao M, Lu B, Fisher LW, Robey PG, et al. SHED: stem cells from human exfoliated deciduous teeth. Proc Natl Acad Sci U S A 2003;100:5807-12.

10. Longa EZ, Weinstein PR, Carlson S, Cummins R. Reversible middle cerebral artery occlusion without craniectomy in rats. Stroke 1989;20:84-91.

11. Leker RR, Gai N, Mechoulam R, Ovadia H. Drug-induced hypothermia reduces ischemic damage: effects of the cannabinoid HU-210. Stroke 2003;34:2000-6.

12. Ginsberg MD. Adventures in the pathophysiology of brain ischemia: penumbra, gene expression, neuroprotection: the 2002 Thomas Willis Lecture. Stroke 2003;34:214-23.

13. Leach MJ, Swan JH, Eisenthal D, Dopson M, Nobbs M. BW619C89, a glutamate release inhibitor, protects against focal cerebral ischemic damage. Stroke 1993;24:1063-7.

14. Altman J. Autoradiographic and histological studies of postnatal neurogenesis. IV. Cell proliferation and migration in the anterior forebrain, with special reference to persisting neurogenesis in the olfactory bulb. J Comp Neurol 1969;137:433-57.

15. Kaplan MS. H,inds JW. Neurogenesis in the adult rat: electron microscopic analysis of light radioautographs. Science 1977;197:1092-4.

16. Doetsch F, Alvarez-Buylla A. Network of tangential pathways for neuronal migration in adult mammalian brain. Proc Natl Acad Sci U S A 1996;93:14895-900.

17. Arvidsson A, Collin T, Kirik D, Kokaia Z, Lindvall O. Neuronal replacement from endogenous precursors in the adult brain after stroke. Nat Med 2002;8:963-70.

18. Zhang R, Zhang Z, Wang L, Wang Y, Gousev A, Zhang L, et al. Activated neural stem cells contribute to stroke-induced neurogenesis and neuroblast migration toward the infarct boundary in adult rats. J Cereb Blood Flow Metab 2004;24:441-8.

19. Wang YQ, Guo X, Qiu MH, Feng XY, Sun FY. VEGF Overexpression Enhances Striatal Neurogenesis in Brain of Adult Rat After a Transient Middle Cerebral Artery Occlusion. J Neurosci Res 2007;85:73-82.

20. Jin K, Zhu Y, Sun Y, Mao XO, Xie L, Greenberg DA. Vascular endothelial growth 
factor (VEGF) stimulates neurogenesis in vitro and in vivo. Proc Natl Acad Sci U S A 2002;99:11946-50.

21. Fan Y, Shen F, Frenzel T, Zhu W, Ye J, Liu J, et al. Endothelial progenitor cell transplantation improves long-term stroke outcome in mice. Ann Neurol 2010;67:488-97.

22. Shyu WC, Lin SZ, Yen PS, Su CY, Chen DC, Wang HJ, et al. Stromal cell-derived factor-1 alpha promotes neuroprotection, angiogenesis, and mobilization/homing of bone marrow-derived cells in stroke rats. J Pharmacol Exp Ther 2008;324:834-49.

23. Arora V, Arora P, Munshi AK. Banking stem cells from human exfoliated deciduous teeth (SHED): saving for the future. J Clin Pediatr Dent 2009;33:289-94.

24. Pachter JS, de Vries, HE, Fabry Z. The blood-brain barrier and its role in immune privilege in the central nervous system. J Neuropathol Exp Neurol 2003;62:593-604.

25. Burns TC, Verfaillie CM, Low WC. Stem cells for ischemic brain injury: a critical review. J Comp Neurol. 2009;515:125-44.

26. Pierdomenico L, Bonsi L, Calvitti M, Rondelli D, Arpinati M, Chirumbolo G, et al. Multipotent mesenchymal stem cells with immunosuppressive activity can be easily isolated from dental pulp. Transplantation 2005;80:836-42.

\section{Figure legends}

Fig. 1. (A) Overhead and (B) coronal views of the injection site. (C) The peri-infarct area. Peri-infarct area (gray), infarct core (black). (D) DiI-labeled transplanted SHED (showed by red) migrated from the original injection site to the peri-infarct area in the cortex and striatum. $(\mathrm{E}, \mathrm{F}, \mathrm{G}, \mathrm{H})$ Hight magnification images of DiI-labeled transplanted SHED in D.

Fig. 2. Motor disability test following injection of SHED and PBS on day 1, 3, 6, 9, 12, and 15. $* P<0.05$. Data are expressed as means \pm SD for three determinations, Student's $t$ - test.

Fig. 3. Infarct area on day 16. Reduction in the infarct volume 16 days after injection of 
SHED (A). Infarct area (described with white outline) on day 16 following injection (B:PBS, C:SHED). Data are expressed as means \pm SDs for three determinations (A). $* P<$ 0.005 , vs. control. Student's $t$-test.

Fig. 4. Doublecortin-positive cells (green: A, B), Neurofilament-positive cells (green: C, D), NeuN-positive cells (green: E, F) and RECA1-positive cells (green: G, H). SHED transplantation group (red) on the ipsilateral side (A, C, E, G) on day 16. PBS group (B, D, $\mathrm{F}, \mathrm{H})$ on day 16 . Bar $=20 \mu \mathrm{m}$.

Fig. 5. VEGF-positive cells (green: A), SDF-1-positive cells (green: D). SHED cells (red: $\mathrm{B}, \mathrm{E}) . \mathrm{C}$ and $\mathrm{F}$ is marge with positive cells and SEHD. Bar $=50 \mu \mathrm{m}(\mathrm{A}-\mathrm{F})$.

Fig. 6. The migration of NPCs from the SVZ to the peri-infarct area on day 16 (A). PBS group (B). Bar $=100 \mu \mathrm{m}(\mathrm{A}, \mathrm{B})$ and $10 \mu \mathrm{m}(\mathrm{C}, \mathrm{D})$. IA, infarct area. Statistical analyses of the density of NPCs (E). Data are expressed as means \pm SDs for three determinations (E). $* P<0.01$, vs. PBS group. Student's $t$-test. 
Fig1.

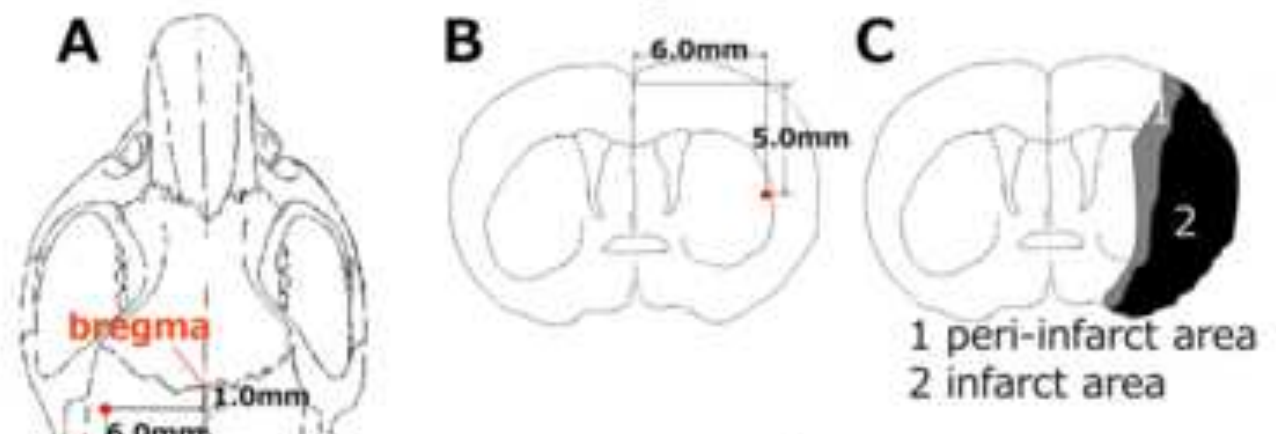

D

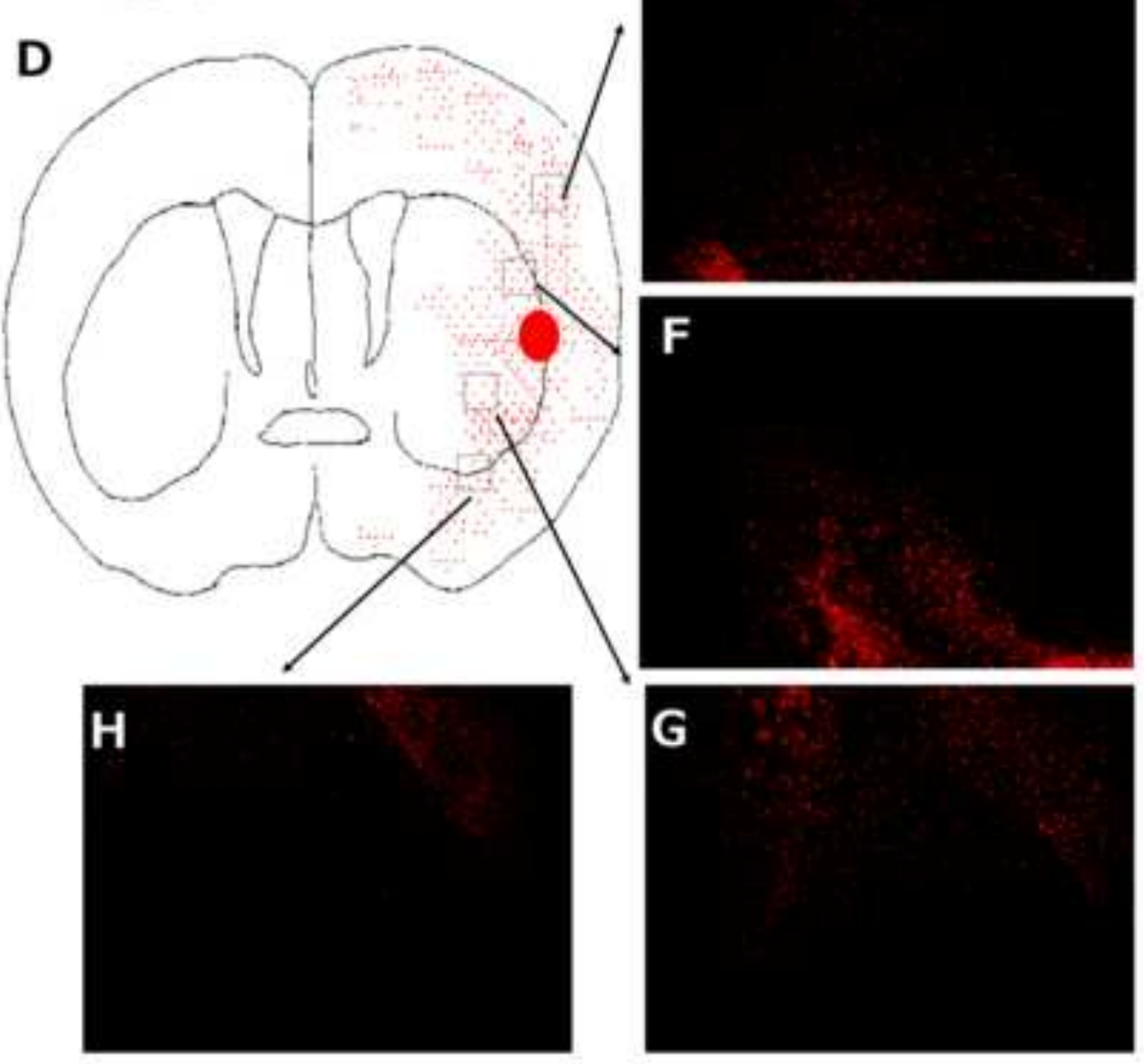


Fig2.

A

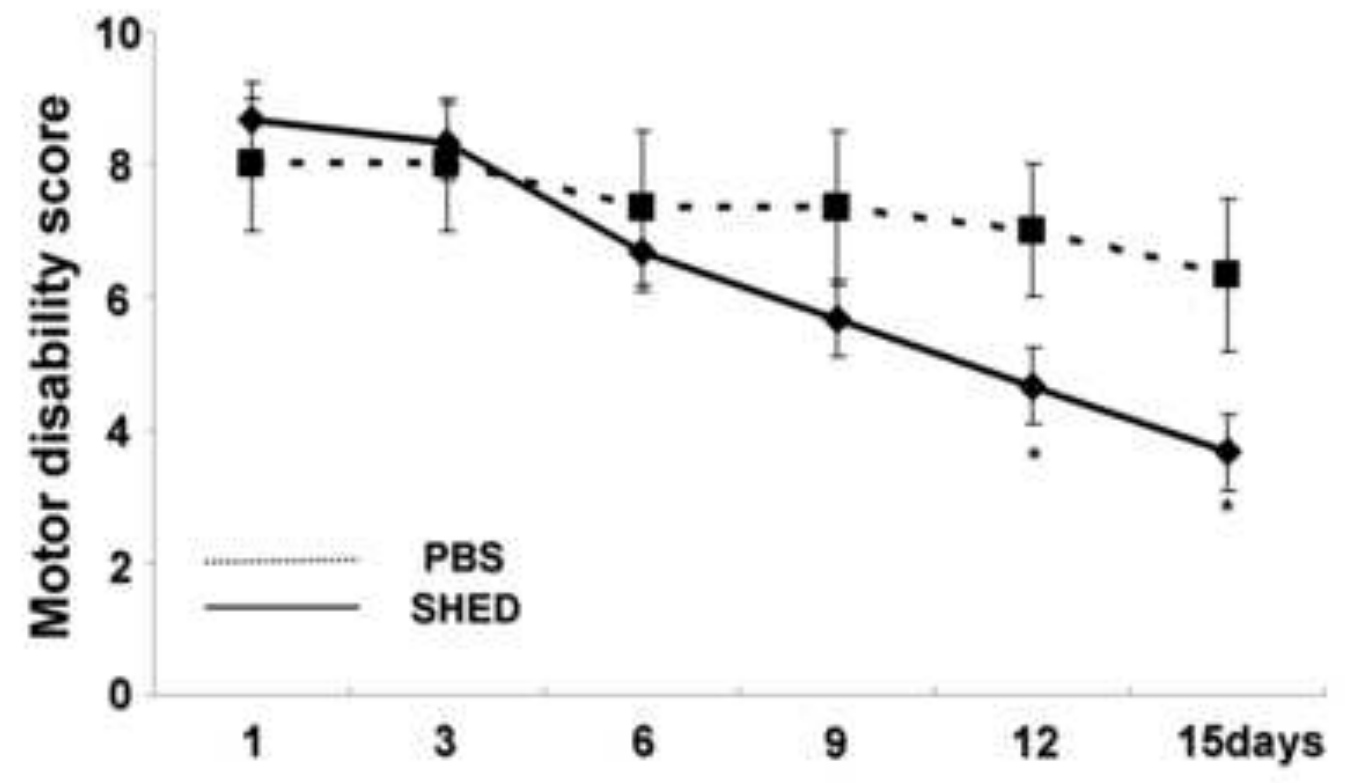


Fig 3.

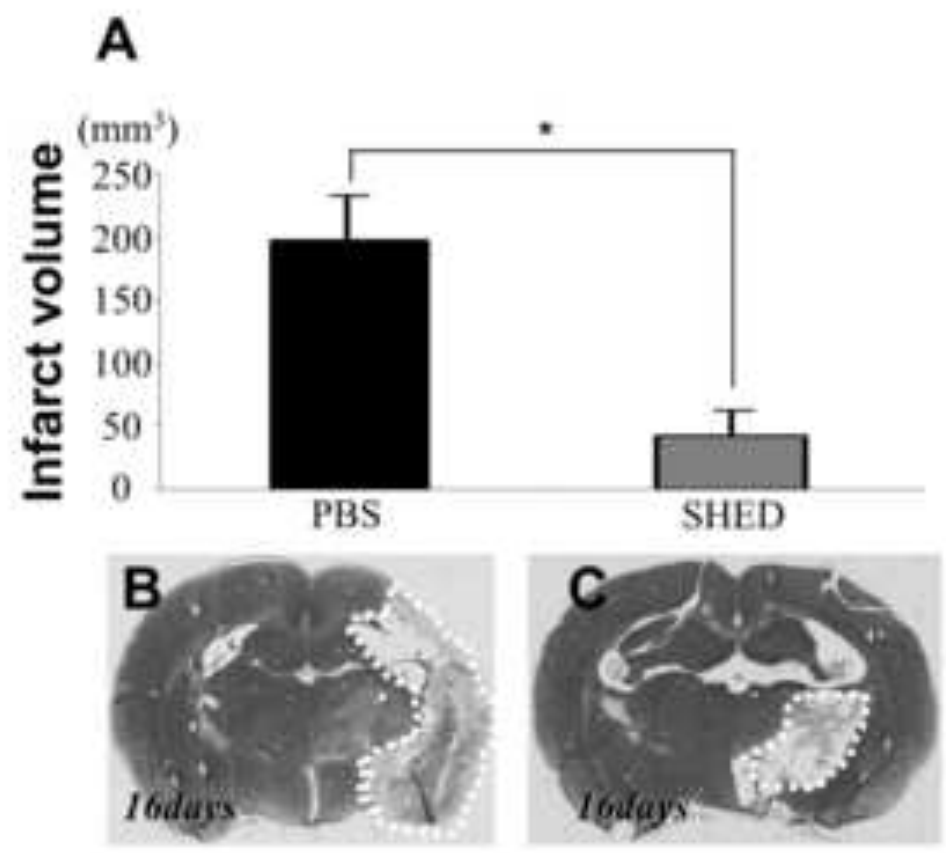


Fig4
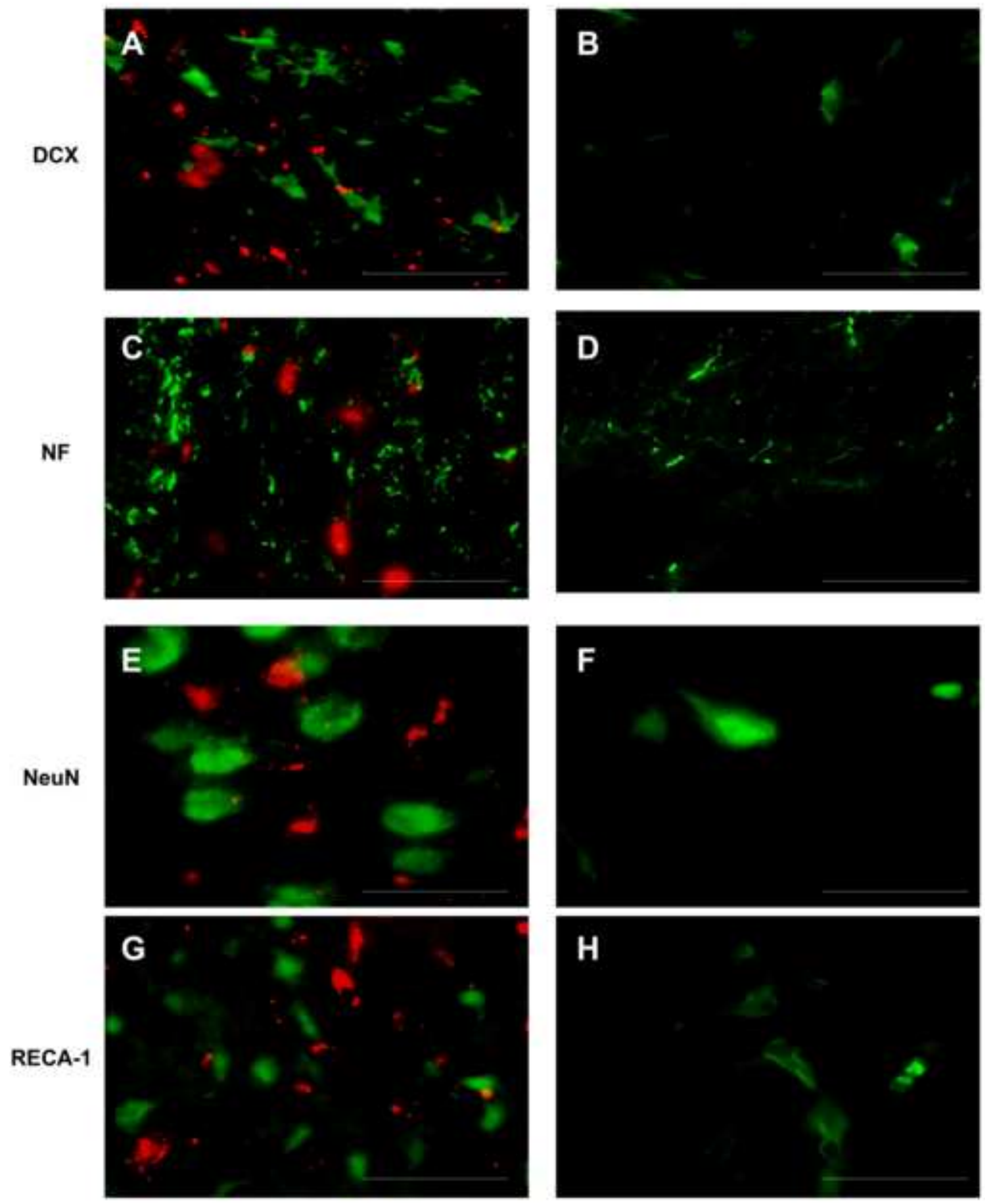

\section{H}


Fig5
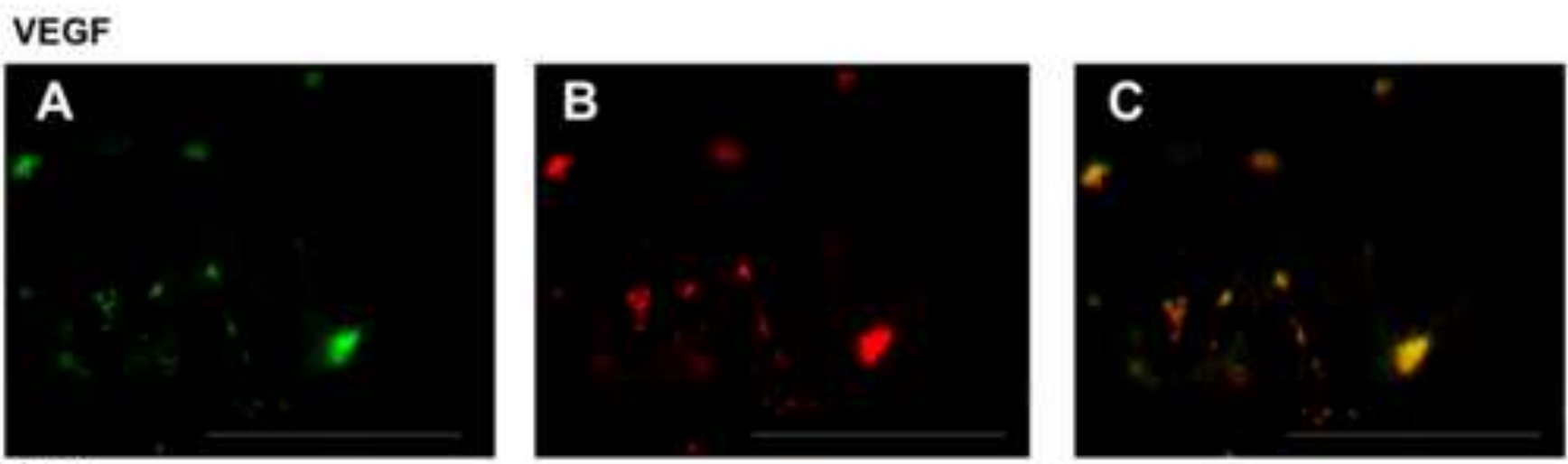

SDF-1
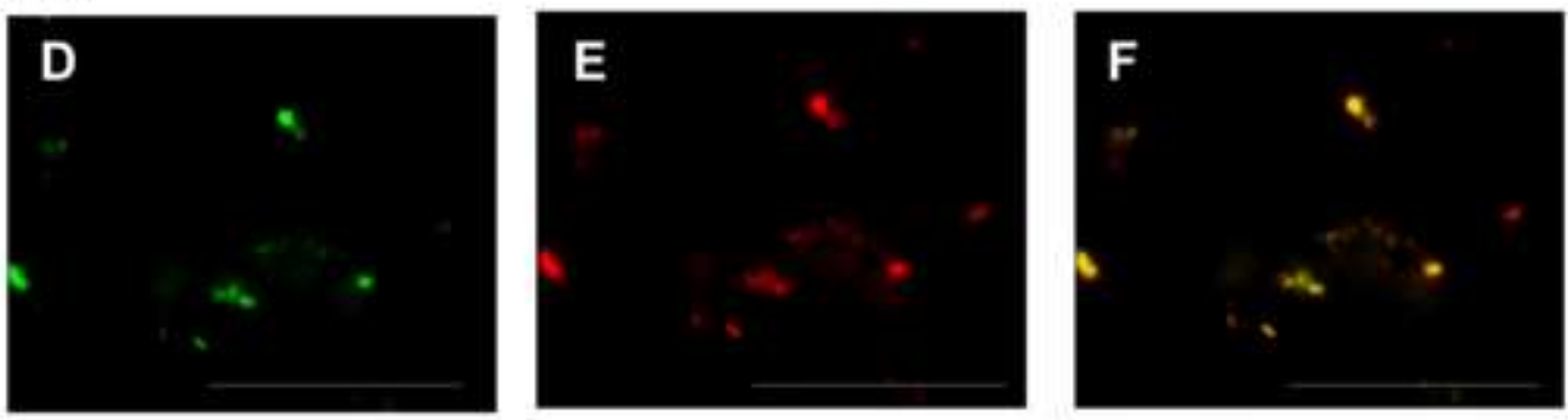
Fig6.

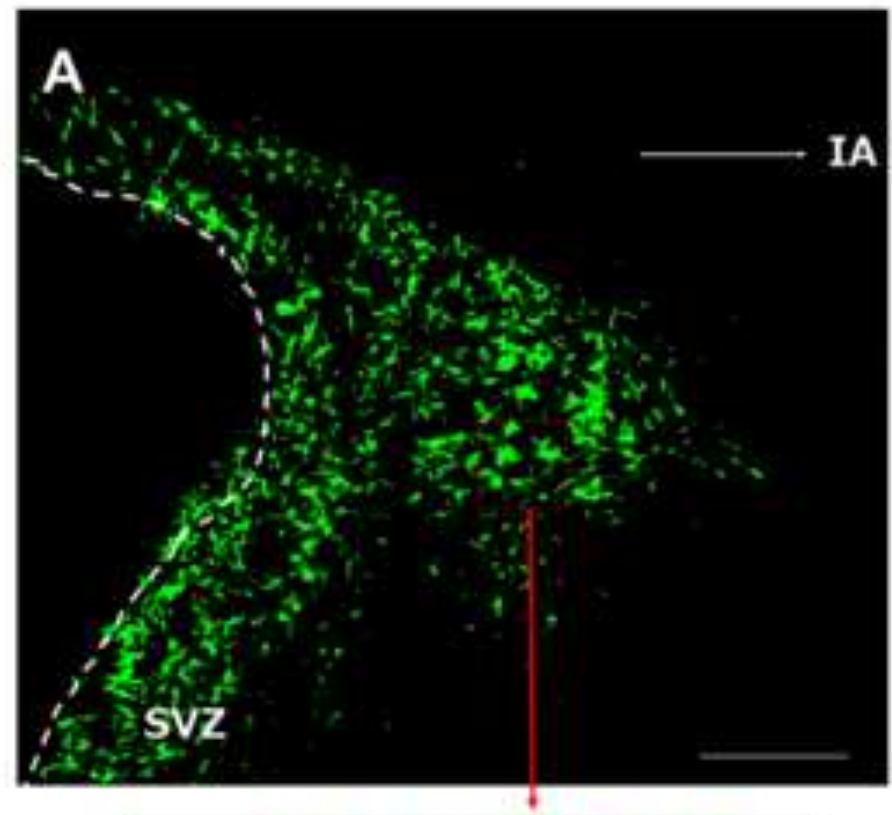

B
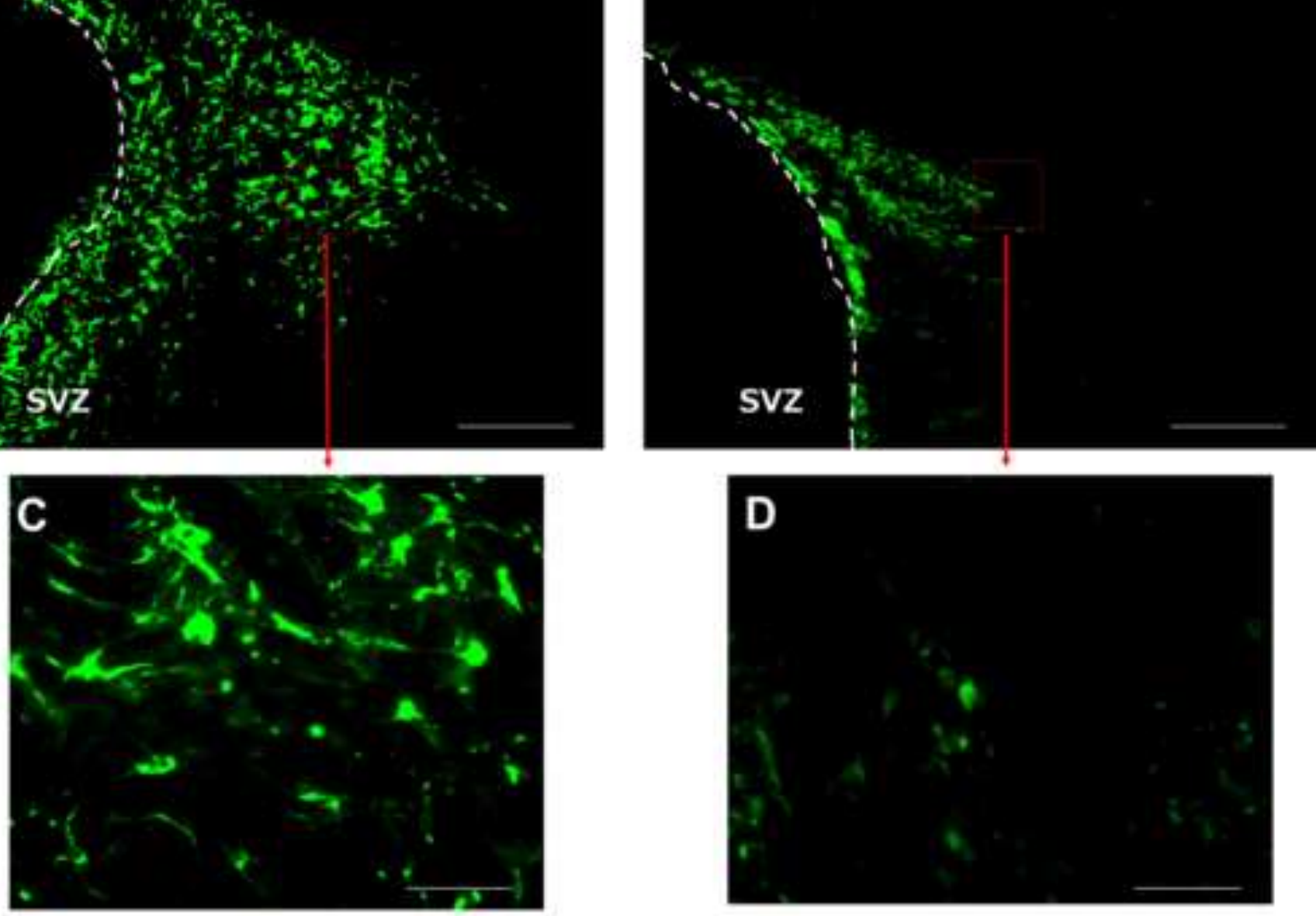

D
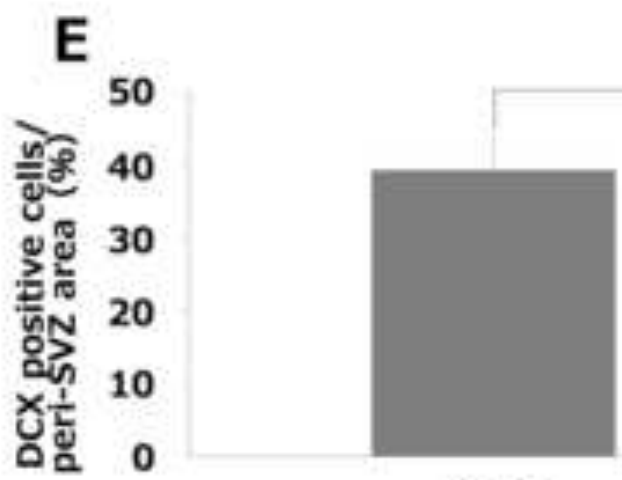

*

\section{SHED}

PBS 